\title{
PARABOLICALLY CONNECTED SUBGROUPS
}

\author{
IGOR V. NETAY
}

\begin{abstract}
We describe all reductive spherical subgroups of the group $\operatorname{SL}(n)$ which have connected intersection with any parabolic subgroup of the group $\operatorname{SL}(n)$. This condition guarantees that any open equivariant embedding of the corresponding homogeneous space into a Moishezon space is algebraic.
\end{abstract}

\section{INTRODUCTION}

Let $G$ be a reductive algebraic group over the field $\mathbb{C}$.

Definition 1. A closed subgroup $H \subset G$ is called parabolically connected if for any parabolic subgroup $P \subseteq G$ the intersection $P \cap H$ is connected.

It is useful to note that an algebraic subgroup $H$ is parabolically connected iff the intersection $B \cap H$ with any Borel subgroup $B$ is connected. Indeed, let $P \subseteq G$ be a parabolic subgroup and $B \subseteq G$ be a Borel subgroup contained in $P$. Then $B$ is also a Borel subgroup of $P$. The connected algebraic group $P$ is a union of its Borel subgroups [1, ch. 8, $\S 22]$, hence $H \cap P=\bigcup_{B \subseteq P}(H \cap B)$. Since any intersection $H \cap B$ is connected and contains the identity element, we obtain that $H \cap P$ is connected.

Since any subgroup of a unipotent group is connected, we see that any unipotent subgroup $H \subset G$ is parabolically connected. It was proved by Hausen [2, Thm. 3] that for any reductive group $H$ the diagonal $\Delta H=\{(h, h): h \in H\}$ is parabolically connected as a subgroup of $G=H \times H$.

Recall that an algebraic subgroup $H \subset G$ is said to be a spherical subgroup if the induced action of some Borel subgroup $B$ in $G$ on the homogeneous space $G / H$ has an open orbit. The main result of this paper is the classification of parabolically connected reductive spherical subgroups in the group $\mathrm{SL}(n)$. Our goal is to choose parabolically connected subgroups in the list of all spherical subgroups [3]. Denote by $\mathrm{S}(\mathrm{GL}(m) \times \mathrm{GL}(n))$ the subgroup of $\mathrm{SL}(m+n)$ which consists of all block matrices with blocks of sizes $m$ and $n$. Denote by $\mathrm{T}^{1}$ the one-dimensional algebraic torus.

Key words and phrases. reductive group, parabolic subgroup, spherical subgroup, flag, Moishezon space. The author is partially supported by AG Laboratory HSE, RF government grant, ag. 11.G34.31.0023. 
Theorem 1. The subgroups

$$
\begin{gathered}
\mathrm{SL}(\mathrm{m}) \times \mathrm{SL}(n) \subset \mathrm{SL}(m+n) \text { for all } m n \\
\mathrm{~S}(\mathrm{GL}(m) \times \mathrm{GL}(n)) \subset \mathrm{SL}(m+n) \text { for } m \neq n, \\
\mathrm{Sp}(2 n) \subset \mathrm{SL}(2 n), \operatorname{Sp}(2 n) \subset \mathrm{SL}(2 n+1) \quad \mathrm{Sp}(2 n) \times \mathrm{T}^{1} \subset \mathrm{SL}(2 n+1)
\end{gathered}
$$

are parabolically connected. At the same time the subgroups

$$
\mathrm{SO}(n) \subset \mathrm{SL}(n) \quad \mathrm{S}(\mathrm{GL}(n) \times \mathrm{GL}(n)) \subset \mathrm{SL}(2 n)
$$

are not parabolically connected.

The notion of parabolically connected subgroup becomes important in complex analysis. Let $X$ be an analytic compact connected complex variety. Denote by $\mathcal{M}(X)$ the field of global meromorphic functions on $X$. It was shown that the transcendence degree of $\mathcal{M}(X)$ is less than or equal to the dimension of $X$. Those varieties for which the equality holds are called Moishezon varieties. It is known that the connected component of the identity in the group of automorphisms $\operatorname{Aut}^{\circ}(X) \subset \operatorname{Aut}(X)$ of a Moishezon space $X$ has a natural structure of affine algebraic group. An action of a connected reductive group $G$ on a Moishezon space $X$ is called algebraic if the corresponding homomorphism $G \rightarrow \operatorname{Aut}^{\circ}(X)$ is a homomorphism of algebraic groups. It is natural to conjecture that if the group $\operatorname{Aut}^{\circ}(X)$ is "sufficiently large", then the space $X$ is an algebraic manifold. The first result is this direction was obtained in the paper [4] by D. Luna.

Theorem 2. [4, Thm. 1] Let $X$ be a Moishezon space equipped with an action of an algebraic torus $\mathrm{T}$ with an open orbit. Then $X$ is an algebraic $\mathrm{T}$-manifold.

The following result by J. Hausen generalizes Theorem 2 .

Theorem 3. [2, Thm. 2] Let $X$ be a compact Moishezon space, and suppose that a reductive group $G$ acts on $X$ algebraically. If for some Borel subgroup $B$ of $G$ the orbit $B \cdot x_{0}$ is dense in $X$ and each closed $G$-orbit contains a point $x$ such that there is a parabolic subgroup $Q \subset G$ opposite to $G_{x}$ with $B \subset Q$ and $G_{x_{0}} \cap Q$ is connected, then $X$ is a complex algebraic $G$-variety.

Corollary 1. Let $H \subset G$ be a spherical parabolically connected subgroup and let $G / H \rightarrow$ $\rightarrow X$ be an open equivariant embedding into Moishezon space $X$ with an action of $G$. Then $X$ is an algebraic $G$-variety. 
In many cases this corollary gives affirmative answer to the question stated in the paper [4]: is it true that any Moishezon space equipped with a locally transitive action of a semisimple simply connected algebraic group $G$ is algebraic $G$-manifold if stabilizer of a point in dense orbit is connected? Example of nonalgebraic PSL(2)-quasihomogeneous Moishezon space is constructed in the paper [5]. It would be interesting to find out if homogenious spaces $\mathrm{SL}(n) / H$ possess open equivariant embeddings into nonalgebraic Moishezon spaces where $H$ is one of two non parabolically connected reductive spherical subgroups in $\mathrm{SL}(n)$.

The final version is to be published in Mat. Sb. [6].

The author is grateful to scientific adviser I.V. Arjantsev for constant attention to this work.

\section{LEMMAS ON COMPATIBLE BASES}

Some statements about existence of appropriate bases will be useful to treat intersection of the subgroup $H$ and Borel subgroups. These results may be interesting themselves.

Denote the full flag $\left\{0 \subset V_{1} \subset \cdots \subset V_{n}=V\right\}$ in a vector space $V$ by the symbol $V_{\bullet}$.

Definition 2. We say that the basis $\left\{e_{1}, \ldots, e_{n}\right\}$ of the space $V$ is compatible with the flag $V_{\bullet}$, if any subspace $V_{i}$ is spanned by some subset of this basis.

Definition 3. We say that the basis $\left\{e_{1}, \ldots, e_{n}\right\}$ of the space $V$ is compatible with the decomposition $V=U \oplus W$, if any vector $e_{i}$ lays in one of the spaces $U$ and $W$.

Definition 4. The hyperbolic basis w. r. t. the skew-symmetric form $\omega$ is the bases $\left\{e_{1}, \ldots, e_{n}\right\}$ such that for all $e_{i}$ the equation $\omega\left(e_{i}, \cdot\right) \equiv 0$ holds or there exists an unique vector $e_{j}$ such that $\omega\left(e_{i}, e_{j}\right)= \pm 1$.

Definition 5. Let $V$ • be a flag in the space $V$ and $W \subset V$ be a subspace. Then the quotient flag $V_{\bullet} / W$ is the flag in $V / W$ that consists of quotient spaces $V_{i} /\left(V_{i} \cap W\right)$.

Lemma 1. Let $V=U \oplus W$ and $V$. be a full flag in the space $V$. Then there exist bases $\left\{e_{1}, \ldots, e_{n}\right\}$ and $\left\{v_{1}, \ldots, v_{n}\right\}$ of the space $V$ such that the basis $\left\{e_{1}, \ldots, e_{n}\right\}$ is compatible with the decomposition $V=U \oplus W$, the basis $\left\{v_{1}, \ldots, v_{n}\right\}$ is compatible with the flag $V$. and each vector $v_{i}$ equals some vector $e_{l}$ or the sum $e_{j}+e_{k}$ of some $e_{j} \in U$ and $e_{k} \in W$.

Proof. Let us construct the bases by induction. On the $k$-th step we construct the basis $\left\{v_{1}, \ldots, v_{k}\right\}$ of the space $V_{k}$ and the basis $\left\{e_{1}, \ldots, e_{l}\right\}$ of the space $\operatorname{pr}_{U}\left(V_{k}\right) \oplus \operatorname{pr}_{W}\left(V_{k}\right)$ which is compatible with the decomposition, where $\operatorname{pr}_{U}$ and $\operatorname{pr}_{W}$ are the projections onto $U$ and $W$ along $W$ and $U$ respectively. Suppose that $k$ steps of the construction are 
done. Let us do the $(k+1)$-st step. Note that for $i=1, \ldots, n$ the equation $\operatorname{dim}\left(V_{i}\right)=$ $=\operatorname{dim}\left(V_{i} \cap U\right)+\operatorname{dim}\left(\operatorname{pr}_{W}\left(V_{i}\right)\right)=\operatorname{dim}\left(V_{i} \cap W\right)+\operatorname{dim}\left(\operatorname{pr}_{U}\left(V_{i}\right)\right)$ holds. Exactly one of the following four cases holds:

$$
\begin{gathered}
\operatorname{dim}\left(\operatorname{pr}_{U}\left(V_{k+1}\right)\right)=\operatorname{dim}\left(\operatorname{pr}_{U}\left(V_{k}\right)\right)+1 \\
\operatorname{dim}\left(\operatorname{pr}_{W}\left(V_{k+1}\right)\right)=\operatorname{dim}\left(\operatorname{pr}_{W}\left(V_{k}\right)\right)+1
\end{gathered}
$$

Then there exists a vector $v \in V_{k+1}$ such that $\operatorname{pr}_{U}(v) \notin \operatorname{pr}_{U}\left(V_{k}\right), \operatorname{pr}_{W}(v) \notin \operatorname{pr}\left(V_{k}\right)$. Let us determine $v_{k+1}=v, e_{l+1}=\operatorname{pr}_{U}(v), e_{l+2}=\operatorname{pr}_{W}(v)$.

$$
\begin{gathered}
\operatorname{dim}\left(\operatorname{pr}_{U}\left(V_{k+1}\right)\right)=\operatorname{dim}\left(\operatorname{pr}_{U}\left(V_{k}\right)\right)+1, \\
\operatorname{dim}\left(U \cap V_{k+1}\right)=\operatorname{dim}\left(U \cap V_{k}\right)+1
\end{gathered}
$$

Take any $v_{k+1}=e_{k+1} \in U \cap\left(V_{k+1} \backslash V_{k}\right)$.

$$
\begin{gathered}
\operatorname{dim}\left(\operatorname{pr}_{W}\left(V_{k+1}\right)\right)=\operatorname{dim}\left(\operatorname{pr}_{W}\left(V_{k}\right)\right)+1, \\
\operatorname{dim}\left(W \cap V_{k+1}\right)=\operatorname{dim}\left(W \cap V_{k}\right)+1
\end{gathered}
$$

This case is similar to the previous.

(4)

$$
\begin{gathered}
\operatorname{dim}\left(U \cap V_{k+1}\right)=\operatorname{dim}\left(U \cap V_{k}\right)+1, \\
\operatorname{dim}\left(W \cap V_{k+1}\right)=\operatorname{dim}\left(W \cap V_{k}\right)+1 .
\end{gathered}
$$

Since $V_{k+1} \cap U \subset \operatorname{pr}_{U}\left(V_{k+1}\right)=\operatorname{pr}_{U}\left(V_{k}\right)$, there exists $u \in V_{k}$ such that $\operatorname{pr}_{U}(u) \in$ $\in V_{k+1} \backslash V_{k}$. Analogously, there exists $w \in V_{k}: \operatorname{pr}_{W}(w) \in V_{k+1} \backslash V_{k}$.

In the case $\operatorname{pr}_{U}(w) \in V_{k+1} \backslash V_{k}$, let us determine $v=w$.

In the case $\operatorname{pr}_{W}(u) \in V_{k+1} \backslash V_{k}$, let us determine $v=u$.

Otherwise we say that $v=u+w$. This implies that $v \in V_{k}, \operatorname{pr}_{U}(v) \in V_{k+1} \backslash V_{k}$, $\operatorname{pr}_{W}(v) \in V_{k+1} \backslash V_{k}$. The vector $v$ can be considered as a linear combination of the basis $\left\{v_{1}, \ldots, v_{k}\right\}: v=\sum_{i=1}^{k} \alpha_{k} v_{k}$. Let us denote

$$
v^{\prime}=\sum_{\substack{i=1, \ldots, k \\ v_{i} \notin U \cup W}} \alpha_{k} v_{k}
$$


Then we have

$$
\begin{aligned}
& \operatorname{pr}_{U}\left(v^{\prime}-v\right)=\sum_{\substack{i=1, \ldots, k \\
v_{i} \in U}} \alpha_{i} v_{i} \in V_{k}, \\
& \operatorname{pr}_{W}\left(v^{\prime}-v\right)=\sum_{\substack{i=1, \ldots, k \\
v_{i} \in W}} \alpha_{i} v_{i} \in V_{k} .
\end{aligned}
$$

Hence,

$$
\begin{gathered}
\operatorname{pr}_{U}\left(v^{\prime}\right) \in V_{k+1} \backslash V_{k}, \quad \operatorname{pr}_{W}\left(v^{\prime}\right) \in V_{k+1} \backslash V_{k}, \\
v^{\prime}=\sum_{\substack{i=1, \ldots, k \\
v_{i} \notin U \cup W}} \alpha_{i} v_{i}=\sum_{\substack{i=1, \ldots, k_{0} \\
v_{i} \notin U \cup U W}} \alpha_{i} v_{i}, \quad k_{0} \leqslant k, \alpha_{k_{0}} \neq 0 .
\end{gathered}
$$

Since $v_{k_{0}} \notin U \cup W$, we have $v_{k_{0}}=e_{s}+e_{t}$ for some $e_{s} \in U, e_{t} \in W$ by the construction. Let us substitute $v_{k_{0}}, e_{s}, e_{t}$ for $v^{\prime}, \operatorname{pr}_{U}\left(v^{\prime}\right), \operatorname{pr}_{W}\left(v^{\prime}\right)$. This substitution is compatible with the flag and the decomposition because $v_{k_{0}} \in V_{k_{0}} \backslash V_{k_{0}-1}$. This shows that required bases for $V_{k+1}$ and $\operatorname{pr}_{U}\left(V_{k+1}\right) \oplus \operatorname{pr}_{W}\left(V_{k+1}\right)$ are constructed.

Lemma 2. Let $V$ be a $2 n$-dimensional vector space with the full flag $V$. and $\omega$ be a nondegenerate skew-symmetric form in the space $V$. Then there exists a basis $\left\{e_{1}, \ldots, e_{2 n}\right\}$ in the vector space $V$ that is compatible with the flag $V_{\bullet}$ and hyperbolic w. r. t. $\omega$.

Proof. The proof is by induction on $n$. The basis of induction is the case $n=1$. Suppose that $V_{1}=\left\langle e_{1}\right\rangle$. Since the form $\omega$ is nondegenerate there exists a vector $e_{2} \in V_{2}=V$ such that $\omega\left(e_{1}, e_{2}\right)=1$. Antisymmetry of the form $\omega$ implies that $e_{2} \notin V_{1}$.

Suppose that inductive hypothesis is proved for $m<n$. Take any vector $e_{1} \in V_{1} \backslash\{0\}$. Determine $k=\min \left\{l:\left.\omega\left(e_{1}, \cdot\right)\right|_{V_{l}} \not \equiv 0\right\}$. Choose a vector $v_{k} \in V_{k}$ such that $\omega\left(v_{1}, v_{k}\right)=$ $=1$. The intersections of the subspaces of the flag $V_{\bullet}$ except $V_{1}$ and $V_{k}$ with the space $\left\langle e_{1}, e_{k}\right\rangle^{\perp}$ form a flag denoted by $V_{\bullet}^{\prime}$. In the space $\left\langle e_{1}, e_{k}\right\rangle^{\perp}$ there is a basis $\left\{e_{2}, \ldots, e_{k-1}, e_{k+1}, \ldots, e_{2 n}\right\}$ compatible with the flag $V_{\bullet}^{\prime}$ and hyperbolic w. r. t. the restriction of the form $\omega$ to $\left\langle e_{1}, e_{k}\right\rangle^{\perp}$. To conclude the proof, it remains to note that the basis $\left\{e_{1}, \ldots, e_{2 n}\right\}$ is as required.

Lemma 3. Let $V$ be a $(2 n+1)$-dimensional vector space, $U \subset V$ be a hyperplane, $V$ • be a full flag in $V$ and $\omega$ be a skew-symmetric form with nondegenerate restriction to $U$. Then in $V$ there is a basis $\left\{e_{1}, \ldots, e_{2 n+1}\right\}$ such that $e_{2 n+1} \in \operatorname{ker}(\omega), e_{1}, \ldots, e_{2 n} \in U$ and each subspace $V_{i}$ is spanned by some vectors $e_{i}, i=1, \ldots, 2 n$ and $e_{i}+e_{2 n+1}, i=1, \ldots, 2 n$ 
and the basis $\left\{e_{1}, \ldots, e_{2 n+1}\right\}$ is hyperbolic with respect to the form $\omega$. Denote $v_{i}=e_{i}$ or $v_{i}=e_{i}+e_{2 n+1}$ in these cases.

Proof. Since the space $V$ is odd-dimensional, the form $\omega$ is degenerate. Suppose that $\operatorname{ker}(\omega)=\left\langle e_{2 n+1}\right\rangle$. The restriction $\left.\omega\right|_{U}$ is nondegenerate, so we have $e_{2 n+1} \notin U$, this means that $V=U \oplus\left\langle e_{2 n+1}\right\rangle$. Let elements of the full flag $U_{\bullet}$ be images of projection pr: $V \rightarrow U$ along $\left\langle e_{2 n+1}\right\rangle$ for elements of the flag $V_{\bullet}$. The application of the previous lemma yields existance of a basis $\left\{u_{1}, \ldots, u_{2 n}\right\}$ that is compatible with the flag $U_{\bullet}$ and hyperbolic w. r. t. $\left.\omega\right|_{U}$. Let vectors $v_{1}, \ldots, v_{2 n}$ be preimages of $u_{1}, \ldots, u_{2 n}$ under the projection pr such that $v_{i} \in V_{i}$. The basis $v_{1}, \ldots, v_{2 n}, e_{2 n+1}$ is compatible with the flag $V_{\bullet}$ and hyperbolic w. r. t. $\omega$, however it may be not compatible with decomposition $V=U \oplus\left\langle e_{2 n+1}\right\rangle$. Since $\operatorname{ker}(\operatorname{pr})=\left\langle e_{2 n+1}\right\rangle$ for any $v$, we have $v-\operatorname{pr}(v) \in\left\langle e_{2 n+1}\right\rangle$. Suppose that $v_{i}-\operatorname{pr}\left(v_{i}\right)=\alpha_{i} e_{2 n+1}$. Consider indexes $i<j$ such that $\omega\left(e_{i}, e_{j}\right)=1$. One of the following four cases holds:

- $\alpha_{i}=0, \alpha_{j}=0$. Let us determine $v_{i}^{\prime}=v_{i}, v_{j}^{\prime}=v_{j}$.

- $\alpha_{i}=0, \alpha_{j} \neq 0$. Let us determine $v_{i}^{\prime}=\alpha_{j} v_{i}, v_{j}^{\prime}=\alpha_{j}^{-1} v_{j}$.

- $\alpha_{i} \neq 0, \alpha_{j}=0$. Let us determine $v_{i}^{\prime}=\alpha_{i}^{-1} v_{i}, v_{j}^{\prime}=\alpha_{i} v_{j}$.

- $\alpha_{i} \neq 0, \alpha_{j} \neq 0$. Let us determine $v_{i}^{\prime}=\alpha_{i}^{-1} v_{i}, v_{j}^{\prime}=\alpha_{i} v_{j}-\alpha_{j} v_{i}$.

Now for $i=1, \ldots, 2 n$ determine $e_{i}=\operatorname{pr}\left(v_{i}^{\prime}\right) \in U$. Thus the basis $\left\{e_{1}, \ldots, e_{2 n+1}\right\}$ is required.

$$
\text { 3. } \operatorname{CASES} \operatorname{SL}(n) \times \mathrm{SL}(m) \subset \mathrm{SL}(m+n) \text { AND } \mathrm{S}(\mathrm{GL}(m) \times \mathrm{GL}(n)) \subset \mathrm{SL}(m+n)
$$

Proposition 1. The subgroup $\mathrm{GL}(m) \times \mathrm{GL}(n) \subset \mathrm{GL}(m+n)$ is parabolically connected.

Proof. The proof is by induction on $(m, n)$ by assumption that $\left(m^{\prime}, n^{\prime}\right) \leqslant(m, n)$ iff $m^{\prime} \leqslant m$ and $n^{\prime} \leqslant n$. The inductive basis for $m=0$ or $n=0$ is equivalent that a Borel subgroup $B \subset \mathrm{GL}$ is connected.

The following obvious remark is needed for the sequel. If $\varphi: G_{1} \rightarrow G_{2}$ is a surjective homomorphism of algebraic groups, the group $G_{2}$ is connected and the group $\operatorname{ker}(\varphi)$ lies in the connected component of the identity of the group $G_{1}^{\circ}$, then the group $G_{1}$ is connected.

Fix a decomposition $V=U \oplus W, \operatorname{dim}(U)=m, \operatorname{dim}(W)=n$ and a full flag $V$ • in the vector space $V$. Denote by $G$ the group $\mathrm{GL}(U) \times \mathrm{GL}(W)$ and by $H$ the group $G \cap \operatorname{Stab}\left(V_{\bullet}\right)$.

Choose a bases $\left\{e_{1}, \ldots, e_{m+n}\right\}$ and $\left\{v_{1}, \ldots, v_{m+n}\right\}$ by Lemma 1. Renumber elements $\left\{e_{1}, \ldots, e_{m+n}\right\}$ to satisfy $e_{1}, \ldots, e_{m} \in U, e_{m+1}, \ldots, e_{m+n} \in W$ and to save the ordering of elements in $U$ and the ordering of elements in $V$. 
Suppose that $v_{1}=e_{1} \in U$ (the case $v_{1}=e_{m+1} \in W$ is similar), $U^{\prime}=U /\left\langle e_{1}\right\rangle$ and $V_{\bullet}^{\prime}=V_{\bullet} /\left\langle e_{1}\right\rangle$. Determine a projection $\varphi: H \rightarrow \operatorname{GL}\left(U^{\prime} \oplus W\right)$. The kernel $\operatorname{ker}(\varphi)$ consists of matrices of the form

$$
\left(\begin{array}{cc|c}
* & * & 0 \\
0 & E & \\
\hline 0 & E
\end{array}\right)
$$

w. r. t. basis $\left\{e_{1}, \ldots, e_{m+n}\right\}$, so it is connected. Then connectivity of $H$ follows from connectivity of the image $\left(\mathrm{GL}\left(U^{\prime}\right) \times \mathrm{GL}(W)\right) \cap \operatorname{Stab}\left(V_{\bullet}^{\prime}\right)$ that is connected by the inductive assumption for $(m-1, n)$.

Suppose that $v_{1}=e_{1}+e_{m+1}, e_{1} \in U, e_{m+1} \in W, U^{\prime}=U /\left\langle e_{1}\right\rangle, W^{\prime}=W /\left\langle e_{m+1}\right\rangle$, the projection $\varphi: H \rightarrow \mathrm{GL}\left(U^{\prime} \oplus W^{\prime}\right)$ and $V_{\bullet}^{\prime}=V_{\bullet} /\left\langle e_{1}, e_{m+1}\right\rangle$. The kernel consists of matrices of the form

$$
\left(\begin{array}{cc|c}
\lambda & * & 0 \\
0 & E & \\
\hline 0 & \lambda & * \\
& 0 & E
\end{array}\right)
$$

and is connected. The image equals $\left(\mathrm{GL}\left(U^{\prime}\right) \times \mathrm{GL}\left(W^{\prime}\right)\right) \cap \operatorname{Stab}\left(V_{\bullet}^{\prime}\right)$. Thus connectivity of $H$ follows from connectivity of the image, i. e. by inductive assumption for $(m-1, n-1)$.

Proposition 2. The subgroup $\mathrm{SL}(m) \times \mathrm{GL}(n) \subset \mathrm{GL}(m+n)$ is parabolically connected.

Proof. Let us prove this proposition as above in the following terms: $G=\mathrm{SL}(U) \times \mathrm{GL}(W) \subset$ $\subset \operatorname{GL}(U \oplus W), H=G \cap \operatorname{Stab}\left(V_{\bullet}\right)$, where $(m, n)=(\operatorname{dim}(U), \operatorname{dim}(W)), V_{\bullet}$ is a full flag in the space $V=U \oplus W$. The proof is by induction on $(m, n)$ with the same ordering. The inductive basis for $m=0$ or $n=0$ is that Borel subgroups SL GL are connected. Choose the bases $\left\{e_{1}, \ldots, e_{m+n}\right\} \quad\left\{v_{1}, \ldots, v_{m+n}\right\}$ and renumber in the same way.

Suppose that $v_{1}=e_{1} \in U, U^{\prime}=U /\left\langle e_{1}\right\rangle, \varphi: H \rightarrow \operatorname{GL}\left(U^{\prime} \oplus W\right), \quad V_{\bullet}^{\prime}=V /\left\langle e_{1}\right\rangle$. The kernel $\operatorname{ker}(\varphi)$ consists of matrices of the form

$$
\left(\begin{array}{cc|c}
1 & * & 0 \\
0 & E & \\
\hline 0 & E
\end{array}\right)
$$

The kernel is connected. The image equals $\left(\mathrm{GL}\left(U^{\prime}\right) \times \mathrm{GL}(W)\right) \cap \operatorname{Stab}\left(V_{\bullet}^{\prime}\right)$ and is connected by Theorem 1 . 
Suppose that $v_{1}=e_{m+1} \in W, W^{\prime}=W /\left\langle e_{m+1}\right\rangle, \quad \varphi: H \rightarrow \operatorname{GL}\left(U \oplus W^{\prime}\right), \quad V_{\bullet}^{\prime}=$ $V_{\bullet} /\left\langle e_{m+1}\right\rangle$. The kernel $\operatorname{ker}(\varphi)$ consists of matrices of the form

$$
\left(\begin{array}{c|cc}
E & \multicolumn{1}{|c}{0} \\
\hline 0 & * & * \\
& 0 & E
\end{array}\right),
$$

so it is connected. The image equals $\left(\mathrm{SL}(U) \times \mathrm{GL}\left(W^{\prime}\right)\right) \cap \operatorname{Stab}\left(V_{\bullet}^{\prime}\right)$ and is connected by the inductive assumption for $(m, n-1)$.

Suppose that $v_{1}=e_{1}+e_{m+1}, e_{1} \in U, e_{m+1} \in W, U^{\prime}=U /\left\langle e_{1}\right\rangle, W^{\prime}=W /\left\langle e_{m+1}\right\rangle$, $\varphi: H \rightarrow \operatorname{GL}\left(U^{\prime} \oplus W^{\prime}\right)$ and $V_{\bullet}^{\prime}=V_{\bullet} /\left\langle e_{1}, e_{m+1}\right\rangle$. The kernel $\operatorname{ker}(\varphi)$ consists of matrices of the form

$$
\left(\begin{array}{cc|cc}
1 & * & 0 \\
0 & E & & \\
\hline 0 & 1 & * \\
& 0 & E
\end{array}\right)
$$

and is connected. The image equals $\left(\mathrm{GL}\left(U^{\prime}\right) \times \mathrm{GL}\left(W^{\prime}\right)\right) \cap \operatorname{Stab}\left(V_{\bullet}^{\prime}\right)$ and is connected by proposition [1, Therefore the group $H$ is connected.

Proposition 3. The subgroup

$$
\{(A, B) \in \mathrm{GL}(m) \times \mathrm{GL}(n): \operatorname{det}(A)=\operatorname{det}(B)\} \subset \mathrm{GL}(m+n)
$$

is parabolically connected.

Proof. We prove this in the same way in the following terms: $G=\{(A, B) \in \mathrm{GL}(U) \times$ $\mathrm{GL}(W): \operatorname{det}(A)=\operatorname{det}(B)\} \subset \operatorname{GL}(U \oplus W), H=G \cap \operatorname{Stab}\left(V_{\bullet}\right)$ where $(m, n)=$ $(\operatorname{dim}(U), \operatorname{dim}(W)), V_{\bullet}$ is a full flag in the space $V=U \oplus W$. The proof is by induction on $(m, n)$ with the same ordering. The inductive basis for $m=0$ or $n=0$ is that Borel subgroups in the group SL are connected. Choose and renumber bases $\left\{e_{1}, \ldots, e_{m+n}\right\}$ and $\left\{v_{1}, \ldots, v_{m+n}\right\}$ in the same way.

Suppose that $v_{1}=e_{1} \in U$ (the case $v_{1}=e_{2 n+1} \in W$ is analogous), $\varphi: H \rightarrow \mathrm{GL}\left(U^{\prime} \oplus\right.$ $W), U^{\prime}=U /\left\langle e_{1}\right\rangle, V_{\bullet}^{\prime}=V_{\bullet} /\left\langle e_{1}\right\rangle$. The kernel $\operatorname{ker}(\varphi)$ consists of elements of the form

$$
\left(\begin{array}{cc|c}
1 & * & 0 \\
0 & E & \\
\hline 0 & E
\end{array}\right) .
$$

This implies that the kernel is connected. The image $\operatorname{Im}(\varphi)$ equals $\left(\mathrm{GL}\left(U^{\prime}\right) \times \mathrm{GL}(W)\right) \cap$ $\cap \operatorname{Stab}\left(V_{\bullet}^{\prime}\right)$ and is connected by proposition 1 . 
Suppose that $v_{1}=e_{1}+e_{m+1}, e_{1} \in U, e_{m+1} \in W, \varphi: H \rightarrow \operatorname{GL}\left(U^{\prime} \oplus W^{\prime}\right), U^{\prime}=U /\left\langle e_{1}\right\rangle$, $W^{\prime}=W /\left\langle e_{m+1}\right\rangle V_{\bullet}^{\prime}=V_{\bullet} /\left\langle e_{1}, e_{m+1}\right\rangle$. The kernel $\operatorname{ker}(\varphi)$ consists of matrices of the form

$$
\left(\begin{array}{cc|c}
\lambda & * & 0 \\
0 & E & \\
\hline 0 & \lambda & * \\
\hline 0 & E
\end{array}\right) .
$$

This yields it is connected. The image $\operatorname{Im}(\varphi)$ equals $\left(\left\{(A, B) \in \operatorname{GL}\left(U^{\prime}\right) \times \operatorname{GL}\left(W^{\prime}\right)\right.\right.$ : $\operatorname{det}(A)=\operatorname{det}(B)\}) \cap \operatorname{Stab}\left(V_{\bullet}^{\prime}\right)$ and is connected by the inductive assumption for $(m-$ $-1, n-1)$.

Therefore the group $H$ is connected.

Proposition 4. The subgroup $\mathrm{SL}(m) \times \mathrm{SL}(n) \subset \mathrm{SL}(m+n)$ is parabolically connected.

Proof. Let us prove that the subgroup $G=\mathrm{SL}(m) \times \mathrm{SL}(n) \subset \mathrm{GL}(m+n)$ is parabolically connected. Let $B \subset \mathrm{GL}(m+n)$ be a Borel subgroup. Then the group $B^{\prime}=B \cap \operatorname{SL}(m+n)$ is a Borel subgroup for $\mathrm{SL}(m+n), \quad G \cap B=G \cap B^{\prime}$, because $G \subset \mathrm{SL}(m+n)$. Hence parabolic connectivity of the group $\operatorname{SL}(n) \times \operatorname{SL}(m)$ as a subgroup of $\operatorname{SL}(m+n)$ is equivalent to parabolic connectivity as a subgroup of $\mathrm{GL}(m+n)$.

The further proof and terms are similar to the previous: $G=\mathrm{SL}(U) \times \mathrm{SL}(W), H=$ $=G \cap \operatorname{Stab}\left(V_{\bullet}\right)$ where $(m, n)=(\operatorname{dim}(U), \operatorname{dim}(W)), V_{\bullet}$ is a full flag in the space $V=$ $=U \oplus W$. The proof is by induction on $(m, n)$ with the same ordering. The inductive basis for $m=0$ or $n=0$ is that Borel subgroups in the group SL are connected. Choose and and renumber bases $\left\{e_{1}, \ldots, e_{m+n}\right\}$ and $\left\{v_{1}, \ldots, v_{m+n}\right\}$ as above.

Suppose that $v_{1}=e_{1} \in U, U^{\prime}=U /\left\langle e_{1}\right\rangle, \varphi: H \rightarrow \operatorname{GL}\left(U^{\prime} \oplus W\right)$ and $V_{\bullet}^{\prime}=V_{\bullet} /\left\langle e_{1}\right\rangle$. One of three following cases holds:

- $\forall i=2, \ldots, m+n \quad \exists j \in\{2, \ldots, m+n\} \quad \exists k: v_{k}=e_{i}+e_{j}$. Denote by $s$ a permutation of the set $\{2, \ldots, m+n\}$ such that for any $i, j, k$ satisfying $e_{i}+e_{j}=v_{k}$ we have $s(i)=j$ and $s(j)=i$. Assume an element $g \in G$ has matrix $\left(a_{i j}\right)$ with respect to the basis $\left\{e_{1} \ldots, e_{m+n}\right\}$. Let us show that $a_{i i}=a_{s(i) s(i)}$ for $i=2, \ldots, m+n$. Fix $i$ and $v_{k}=e_{i}+e_{s(i)}$. For any element $g \in \operatorname{Stab}\left(V_{\bullet}\right)$ we have $g v_{k}=\lambda_{k} v_{k}+v$, $v \in V_{k-1}$. Also, $V_{k}=V_{k-1} \oplus\left\langle v_{k}\right\rangle$ and $V_{k-1} \cap\left\langle e_{i}, e_{s(i)}\right\rangle=0$ by the construction of the basis, so $a_{i i}=a_{s(i) s(i)}=\lambda_{k}$. Suppose that $\left\{k: v_{k} \notin U \cup W\right\}=\left\{i_{1}, \ldots, i_{l}\right\}$. Then $\operatorname{det}\left(\left.g\right|_{U}\right)=a_{11} \lambda_{i_{1}} \ldots \lambda_{i_{l}}=1$ and $\operatorname{det}\left(\left.g\right|_{W}\right)=\lambda_{i_{1}} \ldots \lambda_{i_{l}}$. Therefore $g \in \operatorname{SL}(U) \times \operatorname{SL}(W)$. 
This yields that $a_{11}=1$. Hence, the kernel consists of matrices of the form

$$
\left(\begin{array}{cc|c}
1 & * & 0 \\
0 & E & \\
\hline 0 & E
\end{array}\right),
$$

and is connected. The image equals $\left(\mathrm{SL}\left(U^{\prime}\right) \times \mathrm{SL}(W)\right) \cap \mathrm{Stab}\left(V_{\bullet}^{\prime}\right)$ and is connected by the inductive hypothesis.

- $\exists e_{i}=v_{j} \in U, \nexists i^{\prime}, j^{\prime}: v_{j^{\prime}}=e_{i}+e_{i^{\prime}}$. Then the group $H$ contains the one-dimensional torus $T=\operatorname{diag}\left(\lambda, 1, \ldots, 1, \lambda^{-1}, 1, \ldots, 1\right)$, where $\lambda^{-1}$ equals the $i$-th coordinate. Multiplying by $t$ preimages of all elements of the group $\left(\operatorname{SL}\left(U^{\prime}\right) \times \operatorname{SL}(W)\right) \cap \operatorname{Stab}\left(V_{\bullet}^{\prime}\right)$, we get preimages of all elements for the group $\left(\mathrm{GL}\left(U^{\prime}\right) \times \mathrm{SL}(W)\right) \cap \operatorname{Stab}\left(V_{\bullet}^{\prime}\right)$. As before, the kernel is a unipotent group and then in particalar is connected. The image is connected by proposition 2 ,

- $\exists e_{i}=v_{j} \in W: \exists ! i^{\prime}, j^{\prime}: v_{j^{\prime}}=e_{i}+e_{i^{\prime}}$. It can be assumed that $\operatorname{dim}(U)>1$. In the converse case, $G=\mathrm{SL}(W)$ and the inductive statement is that a Borel subgroup for the group SL is connected. Suppose that $e_{s}+e_{k}=v_{l}$. Then the group $H$ contains the one-dimensional torus $T=\left\{\operatorname{diag}\left(\lambda, 1, \ldots, 1, \lambda^{-1}, 1, \ldots, 1, \lambda^{-1}, 1, \ldots, 1, \lambda, 1, \ldots, 1\right)\right\}$ where the value $\lambda$ coincides with the first and $i$-th coordinates and the value $\lambda^{-1}$ coincides with $s$-th and $k$-th coordinates. As in the previous case, the image equals $\left(\operatorname{GL}\left(U^{\prime}\right) \times \operatorname{SL}(W)\right) \cap \operatorname{Stab}\left(V_{\bullet}^{\prime}\right)$. If there are not $s, k, l$ such that $e_{k}+e_{s}=v_{l}$, then assume $s=2, k>m, k \neq i$. The torus $T$ lies in the group $H$. The kernel and the image are the same to previous.

Suppose that $v_{1}=e_{1}+e_{m+1}, e_{1} \in U, e_{m+1} \in W, U^{\prime}=U /\left\langle e_{1}\right\rangle, W^{\prime}=W /\left\langle e_{m+1}\right\rangle$, $\varphi: H \rightarrow \operatorname{GL}\left(U^{\prime} \oplus W^{\prime}\right), \quad V_{\bullet}^{\prime}=V_{\bullet} /\left\langle e_{1}, e m+1\right\rangle$. We may assume that $\operatorname{dim}(U)>1$ and $\operatorname{dim}(W)>1$. Otherwise the statement is that a Borel subgroup for SL is connected. Then we have either $\exists v_{k}=e_{i}+e_{j}, i>1, j>m+1, e_{i} \in U, e_{j} \in W$ or $\exists v_{k}=e_{i} \in U$ and $\exists v_{l}=e_{j} \in W, i>1, j>m+1$. In both cases the group $H$ contains one-dimensional torus $T=\left\{\operatorname{diag}\left(\lambda, 1, \ldots, 1, \lambda^{-1}, 1, \ldots, 1, \lambda, 1, \ldots, 1, \lambda^{-1}\right)\right\}$ where the value $\lambda$ coincides the first and $(m+1)$-th coordinate, the value $\lambda^{-1}$ coincides $i$-th and $j$-th coordinates. Similarly, the image equals $\left\{(A, B) \in \mathrm{GL}\left(U^{\prime}\right) \times \mathrm{GL}\left(W^{\prime}\right): \operatorname{det}(A)=\operatorname{det}(B)\right\} \cap \operatorname{Stab}\left(V_{\bullet}^{\prime}\right)$. Elements in the kernel of the map $\varphi$ have the form

$$
\left(\begin{array}{cc|c}
1 & * & 0 \\
0 & E & \\
\hline 0 & 1 & * \\
& 0 & E
\end{array}\right),
$$

so the kernel is connected. 
Since the kernel and the image are connected, the group $H$ is connected.

$$
\text { 4. CASE } \mathrm{S}(\mathrm{GL}(m) \times \mathrm{GL}(n)) \subset \mathrm{SL}(m+n), m \neq n
$$

Proposition 5. The subgroup $\mathrm{S}(\mathrm{GL}(m) \times \mathrm{GL}(n)) \subset \mathrm{SL}(m+n)$ is parabolically connected. Proof. The proof is by induction on $(m, n)=(\operatorname{dim}(U), \operatorname{dim}(W))$ in the following terms: $G=\mathrm{S}(\mathrm{GL}(U) \times \mathrm{GL}(W)), H=G \cap \operatorname{Stab}\left(V_{\bullet}\right)$ where $V_{\bullet}$ is a full flag, $V=U \oplus W$. The inductive basis for $m=0$ or $n=0$ is that a Borel subgroup of the group $B \subset \mathrm{SL}$ is connected. As above, choose and renumber bases $\left\{e_{1}, \ldots, e_{m+n}\right\}$ and $\left\{v_{1}, \ldots, v_{m+n}\right\}$.

Suppose that $v_{1}=e_{1} \in U$ (the case $v_{1}=e_{m+1} \in W$ is similar), $U^{\prime}=U /\left\langle e_{e}\right\rangle, \varphi: H \rightarrow$ $\rightarrow \operatorname{GL}\left(U^{\prime} \oplus W\right)$ and $V+\bullet^{\prime}=V_{\bullet} /\left\langle e_{1}\right\rangle$. The kernel $\operatorname{ker}(\varphi)$ consists of matrices of the form

$$
\left(\begin{array}{cc|c}
1 & * & 0 \\
0 & E & \\
\hline 0 & E
\end{array}\right),
$$

and is connected. Since for all $g^{\prime} \in G^{\prime}$ one can choose a matrix element $a_{11}$ of the preimage such that the determinant of element in preimage equals 1 , the image equals $G^{\prime}=\left(\mathrm{GL}\left(U^{\prime}\right) \times \mathrm{GL}(W)\right) \cap \operatorname{Stab}\left(V_{\bullet}^{\prime}\right)$.

Suppose that $v_{1}=e_{1}+e_{m+1}, U^{\prime}=U /\left\langle e_{1}\right\rangle, W^{\prime}=W\left\langle e_{m+1}\right\rangle, \varphi: H \rightarrow \operatorname{GL}\left(U^{\prime} \oplus W^{\prime}\right)$ and $V_{\bullet}^{\prime}=V_{\bullet} /\left\langle e_{1}, e_{m+1}\right\rangle$. The kernel $\operatorname{ker}(\varphi)$ consists of matrices of the form

$$
\left(\begin{array}{cc|c}
1 & * & 0 \\
0 & E & \\
\hline 0 & 1 & * \\
& 0 & E
\end{array}\right)
$$

and is connected. The image equals $\left(\mathrm{GL}\left(U^{\prime}\right) \times \mathrm{GL}\left(W^{\prime}\right)\right) \cap \operatorname{Stab}\left(V_{\bullet}^{\prime}\right)$ and is connected by proposition 1 .

Therefore, connectivity of the kernel and the image implies connectivity of the group $H$.

$$
\text { 5. CASES } \operatorname{Sp}(2 n) \subset \mathrm{SL}(2 n), \operatorname{Sp}(2 n) \subset \mathrm{SL}(2 n+1) \text { AND } \operatorname{Sp}(2 n) \times \mathrm{T}^{1} \subset \mathrm{SL}(2 n+1)
$$

Proposition 6. The subgroup $\operatorname{Sp}(2 n) \subset \mathrm{SL}(2 n+1)$ is parabolically connected.

Proof. Suppose that $V=U \oplus W, \operatorname{dim}(U)=2 n, \operatorname{dim}(W)=1$. Let $\omega$ be a skew-symmetric form on $V$ such that the restriction $\left.\omega\right|_{U}$ is nondegenerate, $W=\operatorname{ker}(\omega), V_{\bullet}$ be a full flag in the space $V$ and $H=\operatorname{Sp}(2 n) \cap \operatorname{Stab}\left(V_{\bullet}\right)$. By Lemma 3 choose a basis $\left\{e_{1}, \ldots, e_{2 n+1}\right\}$ in the space $V$. We can write equations on matrix elements in terms of this basis. Assume 
IGOR V. NETAY

$A=\left(a_{i j}\right) \in \mathrm{SL}(V)$. The condition $A \in H$ is equivalent to the conditions $\left.A\right|_{U} \in \mathrm{SL}(U)$, $A^{t} \Omega A=\Omega, A v_{i} \in V_{i}, i=1, \ldots, 2 n+1$, where $\Omega=\left(\omega\left(e_{i}, e_{j}\right)\right)$. Since $\forall\left(a_{i j}\right) \in \operatorname{Sp}(2 n) \subset$ $\subset \mathrm{SL}(2 n+1)$, we have $a_{2 n+1,2 n+1}=1, a_{i, 2 n+1}=a_{2 n+1, i}=0, i=1, \ldots, 2 n$. Hence we can consider $2 n \times 2 n$-matrices.

Let us introduce the following notation. Denote $I=\{1, \ldots, 2 n\}$,

$$
S=\left\{i: \exists j v_{j}=e_{i}+e_{2 n+1}\right\}
$$

Denote by $I^{b}, I^{\sharp}$ subsets in $I$ such that if $\omega\left(e_{i}, e_{j}\right)=1$, then $i \in I^{b}, j \in I^{\sharp}$. The conditions that the basis $\left\{e_{i}\right\}_{i \in I}$ is hyperbolic and the restriction of the form $\omega$ to the hyperplane $U$ is nondegenerate imply that $I^{b} \sqcup I^{\sharp}=I$. Let us write $i=j^{b}, i^{\sharp}=j$ for shortness. Only one of the expressions $i^{b}$ and $i^{\sharp}$ makes sense. Denote this expression by $\bar{i}$. The symbol $i^{\sharp}$ makes sense only for $i \in I^{b}$ and similarly for $j^{b}$. Now let us obtain a system of equation on elements of the group $H$ :

$$
\left\{\begin{array}{l}
a_{i, 2 n+1}=0, \quad i=1, \ldots, 2 n \\
a_{2 n+1, i}=0, \quad i=1, \ldots, 2 n \\
a_{2 n+1,2 n+1}=1 \\
a_{i, j}=0, \quad i>j \\
\sum_{i \in I^{b}} a_{i, l} a_{i^{\sharp}, m}-\sum_{i \in I^{\sharp}} a_{i, l} a_{i^{\natural}, m}=\omega\left(e_{l}, e_{m}\right), \quad l, m=1 \ldots, 2 n \\
\sum_{i \in S} a_{i, k}= \begin{cases}1, & k \in S \\
0, & k \notin S\end{cases}
\end{array}\right.
$$

Let us show how to obtain these equations. The equations (5.2) - (5.4) are equivalent that $A \in \mathrm{SL}(W)$.

Invariance of the flag $A v_{i} \in V_{i}$ implies that $A v_{i} \in\left\langle e_{1}, \ldots, e_{i}, e_{2 n+1}\right\rangle ; A e_{2 n+1}=e_{2 n+1}$, $v_{i}=e_{i}$ or $v_{i}=e_{i}+e_{2 n+1}$ for $i=1, \ldots, 2 n \Rightarrow A e_{i} \in\left\langle e_{1}, \ldots, e_{i}, e_{2 n+1}\right\rangle$. Combining this and (5.3), we obtain (5.5).

The equations (5.6) are equivalent that $A^{t} \Omega A=\Omega$.

Suppose that $k \in S$. Then we have $A v_{k}=A\left(e_{k}+e_{2 n+1}\right)=A e_{k}+e_{2 n+1} \in$ $\in\left\langle\left\{e_{i}\right\}_{i \notin S, i \leqslant k},\left\{e_{i}+e_{2 n+1}\right\}_{i \in S, i \leqslant k}\right\rangle$. At the same time the equations $A e_{k}=\sum_{i=1}^{k} a_{i, k} e_{i}$, $A e_{2 n+1}=e_{2 n+1}$ hold. This yields that $e_{2 n+1}=\sum_{S \ni i \leqslant k} a_{i, k} e_{2 n+1}$, i. e. $\sum_{S \ni i \leqslant k}^{i=1} a_{i, k}=$ $=1 \Leftrightarrow \sum_{i \in S} a_{i, k}=1$.

By the same arguments for $k \notin S$, we have $\sum_{i \in S} a_{i, k}=0$. 
Now let us conclude from these equations that $A \in \mathrm{SL}(W), A^{t} \Omega A=\Omega, A v_{i} \in V_{i}$ $i=1, \ldots, 2 n+1$. Evidently, the system of equations follows the first and the second conditions. Let us check the third.

Suppose that $k \in S$. Then $A v_{k}=A e_{k}+e_{2 n+1}=\sum_{i=1}^{k} a_{i, k} e_{i}+e_{2 n+1}=\sum_{i=1}^{k} a_{i, k} e_{i}+$ $+\sum_{S \ni i \leqslant k} a_{i, k} e_{2 n+1}=\sum_{i=1}^{k} a_{i, k} v_{i} \in V_{i}$. The case $k \notin S$ is similar.

We prove the proposition by induction. The subgroup $\operatorname{SL}(2) \subset \operatorname{SL}(3)$ is parabolically connected by proposition 4. The inductive assumption is that the subgroup $\operatorname{Sp}(2 n-2) \subset$ $\subset \mathrm{SL}(2 n-1)$ is parabolically connected. Let us show that the subgroup $\operatorname{Sp}(2 n) \subset \operatorname{SL}(2 n+$ $+1)$ is parabolically connected. Denote by $L$ the vector space $\left\langle e_{1}, e_{1^{\sharp}}\right\rangle$. Consider the homomorphism $\varphi: H \rightarrow \operatorname{GL}(V / L)$, where $H=\operatorname{Sp}(2 n) \cap \operatorname{Stab}\left(V_{\bullet}\right)$. We shall see that its kernel is connected. Suppose that $\left(a_{i j}\right) \in \operatorname{ker}(\varphi)$. Then the matrix $\left(a_{i j}\right)$ has the form

$$
\left(\begin{array}{cccc}
\lambda & * & \mu & * \\
0 & E & * & 0 \\
0 & 0 & \lambda^{-1} & * \\
0 & 0 & 0 & E
\end{array}\right) .
$$

By equation (5.6) we obtain that elements denoted by $*$ equals 0 . Either some subspace $V_{k}$ contains $e_{1}+e_{2 n+1}$ and does not contain $e_{1}$ or contains $e_{1^{\sharp}}+e_{2 n+1}$ and does not contain $e_{1 \sharp}$. Then for all $\left(a_{i j}\right) \in \operatorname{ker}(\varphi)$ we have $a_{11}=a_{1^{\sharp} 1^{\sharp}}=\lambda=1$. Otherwise the value $\lambda$ may be any in $\mathbb{C}^{\times}$. Thus the group is unipotent or equals semidirect product of one-dimensional torus and an unipotent group. Therefore the kernel $\operatorname{ker}(\varphi)$ is connected. Clearly, the image lies in $\operatorname{Sp}(2 n-2) \cap \operatorname{Stab}\left(V_{\bullet} / L\right)$. Let us show that the image equals $\operatorname{Sp}(2 n-2) \cap \operatorname{Stab}\left(V_{\bullet} / L\right)$. Suppose that $\varphi\left(e_{i}\right)=e_{i}^{\prime}$. Then the set $\left\{e_{i}^{\prime}\right\}_{i \neq 1,1 \sharp}$ is a basis of $V / L$. Let $\left(a_{i j}^{\prime}\right)$ be a matrix of element $A^{\prime} \in \operatorname{Sp}(2 n-2) \cap \operatorname{Stab}\left(V_{\bullet} / L\right)$ w. r. t. this basis. Let $A$ be an element of preimage with the matrix $a_{i j}$ in the basis $\left\{e_{i}\right\}$, where $a_{i j}=a_{i j}^{\prime}$ for $\{i, j\} \cap\left\{1,1^{\sharp}\right\}=\varnothing, a_{11}=a_{1^{\sharp} 1^{\sharp}}=1$, the rest elements equals 0 . We shall see that $A \in H$. Indeed, the operator $A$ stabilizes the skew-symmetric form $\omega$ and the flag $V_{\bullet}$. Since $A^{\prime}$ stabilizes $V_{i} / L$, the operator $A$ stabilizes $V_{i}$. Since $A$ stabilizes $e_{1}$, the statements $A e_{2 n+1} \in V_{i} / L$ for $e_{2 n+1} \in V_{i} / L$ and $A\left(e_{k}+e_{2 n+1}\right) \in V_{i}$ for $e_{k}+e_{2 n+1} \in V_{i}$, $k=1$ or $1^{\sharp}$ are equivalent. Hence connectivity of the group $\operatorname{Sp}(V / L) \cap \operatorname{Stab}\left(V_{\bullet}^{\prime}\right)$ implies connectivity of the group $H$.

Proposition 7. The group $\mathrm{Sp}(2 n) \subset \mathrm{SL}(2 n)$ is parabolically connected.

Proof. Let us conclude this from parabolic connectivity of $\operatorname{Sp}(2 n) \subset \operatorname{SL}(2 n+1)$. Indeed, assume that the group $\operatorname{Sp}(2 n)$ acts on the hyperplane $U$ in the space $V$. Then connectivity of intersection $\operatorname{Sp}(2 n)$ with stabilizer of any full flag $V$ • implies connectivity of intersection 
with stabilizer of any flag $U_{\bullet} \cup\{V\}$, i. e. connectivity of intersection with any Borel subgroup in $\mathrm{SL}(V)$ implies connectivity of the intersection with any Borel subgroup for $\mathrm{SL}(U)$.

Proposition 8. The subgroup $\mathrm{Sp}(2 n) \times \mathrm{T}^{1} \subset \mathrm{SL}(2 n+1)$ is parabolically connected, where $\left.t(\lambda)\right|_{U}=\lambda$ and $t(\lambda) e_{2 n+1}=\lambda^{-2 n} e_{2 n+1}$ for $\left\{t(\lambda)=\operatorname{diag}\left(\lambda, \ldots, \lambda, \lambda^{-2 n}\right)\right\}=\mathrm{T}^{1}$.

Proof. Suppose that $V=U \oplus W, \operatorname{dim}(W)=1, \operatorname{Sp}(2 n) \subset \operatorname{SL}(U)$. Let $B$ be a Borel subgroup for $\operatorname{SL}(V)$. Determine a homomorphism $\varphi: \operatorname{Sp}(2 n) \times T^{1} \rightarrow \mathbb{C}^{\times}, \varphi(A)=\left.A\right|_{W}$. By Lemma 3 choose a basis $\left\{e_{1}, \ldots, e_{2 n+1}\right\}$ in the space $V$. In notation of proposition 6 for each pair $(i, \bar{\imath}), i \in S$ determine $t_{i}=\lambda^{-n}, t_{\bar{\imath}}=\lambda^{n+1}, t_{2 n+1}=\lambda^{-n}$, where the set $S$ is determined by formula (5.1). If $i, \bar{\imath} \notin S$, then determine $t_{i}$ similarly to the case $i \in S$ or $\bar{\imath} \in S$. Then $\operatorname{diag}\left(t_{1}, \ldots, t_{2 n+1}\right) \in\left(\operatorname{Sp}(2 n) \times T^{1}\right) \cap B$. Hence the image $\varphi\left(\operatorname{Sp}(2 n) \times T^{1} \cap B\right)$ equals $\mathbb{C}^{\times}$. The kernel equals $\operatorname{Sp}(2 n) \cap B$ and is connected by proposition 6 , Therefore for any Borel subgroup $B \subset \mathrm{SL}(V)$ the intersection $B \cap\left(\operatorname{Sp}(2 n) \times T^{1}\right)$ is connected.

This completes the proof of Theorem 1 .

\section{LACK OF PARABOLIC CONNECTIVITY}

Assume that the group $\mathrm{SO}(n), n \geqslant 2$ stabilizes the standard symmetric form. Then its intersection with the group of superdiagonal matrices is finite and therefore is not connected. This shows that the subgroup $\mathrm{SO}(n) \subset \mathrm{SL}(n)$ is not parabolically connected.

Proposition 9. The subgroup $\mathrm{S}(\mathrm{GL}(n) \times \mathrm{GL}(n)) \subset \mathrm{SL}(2 n)$ is not parabolically connected.

Proof. Let $\left\{e_{1}, \ldots, e_{n}\right\}$ be a basis in the space $U$ and $\left\{e_{n+1}, \ldots, e_{2 n}\right\}$ be a basis in the space $W, V_{i}=V_{i-1} \oplus\left\langle e_{i}+e_{n+i}\right\rangle, i=1, \ldots, n, V_{0}=0, V_{n+i}=V_{n+i-1} \oplus\left\langle e_{i}\right\rangle, i=1, \ldots, n$. Consider the group $H=\mathrm{S}(\mathrm{GL}(U) \times \mathrm{GL}(W)) \cap \operatorname{Stab}\left(V_{\bullet}\right)$. Take an arbitrary element $g \in H$ and consider its matrix $\left(a_{i j}\right)$ with respect to the basis $\left\{e_{1}, \ldots, e_{2 n}\right\}$. Sice $g\left(e_{i}+e_{n+i}\right) \in V_{i}$, we have $a_{i i}+a_{i, n+i}=a_{n+i, i}+a_{n+i, n+i}$, at the same time $a_{i, n+i}=a_{n+i, i}=0$ by invariance of the subspaces $U$ and $W$. Hence we have $a_{i i}=a_{n+i, n+i}=\lambda_{i}$ for $i=1, \ldots, n$. It follows that $\lambda_{1}^{2} \ldots \lambda_{n}^{2}=\operatorname{det}(g)=1$, i. e. $\lambda_{1} \ldots \lambda_{n}= \pm 1$. The group consists of two non-intersecting nonempty closed subsets. This means that the group $H$ is not connected.

\section{REFERENCES}

1. J.E. Humphreys, Linear Algebraic Groups (Springer-Verlag, Berlin, 1975)

2. J. Hausen, Algebraicity criteria for almost homogeneous complex spaces. Arch. Math. 74 (2000), 317320.

3. M. Krämer, Sphärische untergruppen in kompakten zusammenhängenden Liegruppen. Compositio Math. 38:2 (1979), 129-153. 
4. D. Luna, Toute variété de Moisezon presque homogène sous un tore est un schéma. C. R. Acad, Sci. Paris, t. 314, Série I, p. 65-67, 1992.

5. D. Luna, L. Moser-Jauslin, Th. Vust, Almost homogeneous Artin-Moishezon varieties under the action of $\mathrm{PSL}_{2}(\mathbb{C})$. Topological methods in algebraic transformation groups (New Brunswick, NJ, 1988), 107-115, Progr. Math. 80, Birkhäuser Boston, MA, 1989.

6. I. Netay, "Parabolically connected subgroups", to appear in Mat. Sb.

Department of Mechanics and Mathematics, Moscow State University, Moscow, Russia

IndePendent University of Moscow, Moscow, Russia

E-mail address: inetay@hse.ru 